\title{
Prospective noninterventional study on the use of panitumumab monotherapy in patients with recurrent or progressive colorectal cancer: the VECTIS study
}

\author{
Radek Lakomy ${ }^{1,2}$ \\ Wojciech Rogowski ${ }^{3}$ \\ Bela Piko ${ }^{4}$ \\ Zh Mihaylova ${ }^{5}$ \\ Eva Pritzova ${ }^{6}$ \\ Lucia Kvocekova ${ }^{7}$ \\ 'Department of Comprehensive \\ Cancer Care, Masaryk Memorial \\ Cancer Institute, Brno, Czech \\ Republic; ${ }^{2}$ Faculty of Medicine, \\ Masaryk University, Brno, Czech \\ Republic; ${ }^{3}$ Department of \\ Chemotherapy, Warmia and Masuria \\ Oncology Center, Olsztyn, Poland; \\ ${ }^{4}$ Pándy Kálmán County Hospital, \\ Gyula, Hungary; ${ }^{5}$ Department of \\ Medical Oncology, Military Medical \\ Academy, Sofia, Bulgaria; ${ }^{6}$.D. \\ Roosevelt Teaching Hospital with \\ Policlinic, Banska Bystrica, Slovak \\ Republic; ${ }^{7}$ AMGEN s.r.o., Prague, \\ Czech Republic
}

\author{
This article was published in the following Dove Press journal: \\ Cancer Management and Research \\ 23 October 2015 \\ Number of times this article has been viewed
}

Purpose: Epidermal growth factor receptor-targeted monoclonal antibodies are active as monotherapy beyond second-line treatment. Skin toxicities (STs) are common during treatment, and a positive association between ST severity and patient outcome has been reported. This study collected information on panitumumab monotherapy use in patients with KRAS exon 2 wild-type metastatic colorectal cancer in clinical practice.

Methods: This open-label, prospective, observational, noninterventional study included adult patients who had failed prior chemotherapy with 5-fluorouracil, oxaliplatin, and irinotecan. Patients received panitumumab monotherapy ( $6 \mathrm{mg} / \mathrm{kg}$ every 2 weeks) for $\leq 18$ cycles. Effectiveness was assessed as disease control rate (DCR), tumor response, and freedom from progression. The incidence of ST and other adverse drug reactions (ADRs) was recorded, as were Eastern Cooperative Oncology Group performance status (ECOG PS) and quality of life. The KRAS analysis process was also evaluated.

Findings: The full analysis set included 632 patients (64.6\% male; mean age, 62.3 years), who completed a mean of 9.6 panitumumab cycles. ST, mainly grade $1 / 2$, occurred in $84.3 \%$ of patients, $82.7 \%$ of whom required treatment. Nonskin ADRs occurred in $3.5 \%$ of patients. By the end of treatment, the DCR was $58.9 \%$ overall, and was $53.8 \%$ and $62.7 \%$, respectively in patients with ST grade $0 / 1$ and grade $2 / 3$. Significant associations were observed between maximum ST grade and best response $(P=0.0009)$, DCR $(P=0.0046)$, tumor response $(P=0.0002)$, and freedom from progression $(P=0.0084)$. At the end of the study, $67.4 \%$ of the patients had an ECOG PS of 0/1. Quality of life was rated as "very good" or "good" in 70.3\% of patients. Mean time to obtain KRAS results was 18.2 days; satisfaction with different aspects of KRAS testing was "very good" or "good" in $80 \%-97 \%$ of patients.

Conclusion: Panitumumab monotherapy showed adequate effectiveness and safety in patients with heavily pretreated $K R A S$ exon 2 wild-type metastatic colorectal cancer. The most common ADR was grade $1 / 2 \mathrm{ST}$.

Keywords: observational study, panitumumab, metastatic colorectal cancer, skin toxicity, KRAS

\section{Introduction}

Despite improvements in the diagnosis and treatment of metastatic colorectal cancer $(\mathrm{mCRC})$, it remains the second leading cause of cancer-related death in the European Union, with 215,000 deaths occurring in 2012. ${ }^{1}$ Treatment for $\mathrm{mCRC}$ is generally based on combinations of chemotherapy (5-fluorouracil, oxaliplatin, and irinotecan) and targeted drugs (panitumumab, cetuximab, bevacizumab, aflibercept, and regorafenib). ${ }^{2-4}$ 
In patients who have progressed after second-line therapy, however, appropriate treatment options are less clear. While chemotherapy may still be an option for some patients, panitumumab and cetuximab, two monoclonal antibodies (mAbs) targeted against the epidermal growth factor receptor (EGFR), have demonstrated activity as monotherapy beyond the second line of treatment. ${ }^{5}$

Panitumumab is a fully human mAb, of the immunoglobulin class $(\operatorname{IgG} 2 \mathrm{~K})$, which binds specifically to the human EGFR, and was the first fully human anti-EGFR $\mathrm{mAb}$ approved by the European Medicines Agency for the treatment of mCRC. ${ }^{6-8}$ Panitumumab blocks EGFR signaling in tumor cells by forming receptor dimers, through which autophosphorylation of the EGFR by intracellular tyrosine kinase is inhibited. ${ }^{9}$

During the development of the EGFR-targeted mAbs, it soon became apparent that these agents were not effective in patients whose tumors harbored mutations in the $K R A S$ gene. KRAS mutations, particularly in exon 2, were identified as a predictive marker of resistance in patients with mCRC, ${ }^{10-13}$ and practice guidelines issued by the European Society for Medical Oncology stressed that EGFR-targeted mAbs should not be used in patients with tumors harboring such mutations. ${ }^{14}$ More recently, activating mutations in other regions of $K R A S$, as well as in the closely related $N R A S$ gene, were found to be associated with lack of response to EGFRtargeted mAbs. ${ }^{15-21}$ As a result, the product labeling for panitumumab and cetuximab now states that the mutational status of KRAS and NRAS (exons 2-4) should be determined by an experienced laboratory, using validated test methods, ahead of treatment initiation. ${ }^{8,22}$

Skin toxicities (STs), a pharmacological effect related to the mechanism of action of EGFR-targeted agents, appear in most patients treated with panitumumab. Clinical trial data have shown that most skin events are mild to moderate in severity, with a median time to the first symptom of approximately 10 days, and a median time to resolution of approximately 28 days after the last panitumumab dose. ${ }^{8}$

Furthermore, some panitumumab studies have demonstrated a positive association between the severity of STs and patient outcomes in mCRC. ${ }^{23-25}$ STs should not, therefore, be considered a contraindication for the use of therapy.

The present large, multicenter, prospective, noninterventional study assessed the use of Vectibix ${ }^{\circledR}$ (panitumumab) monotherapy in patients with recurrent or progressive colorectal cancer (VECTIS) in routine clinical practice in central and eastern European (CEE) countries. This study was designed to collect information on panitumumab monotherapy, when used in accordance with the European Summary of medicinal Product Characteristics, in daily clinical practice in patients with $K R A S$ wild-type (WT) mCRC. The study was also designed to collect data specifically related to the occurrence of STs and their management in clinical practice, as well as to evaluate practical aspects associated with obtaining tumor samples and the assessment of KRAS status.

\section{Patients and methods Study design and treatment}

VECTIS was an international, open-label, prospective, observational, noninterventional study conducted between December 2008 and July 2013 in six CEE countries: Bulgaria, Czech Republic, Hungary, Poland, Slovakia, and Slovenia. Participating sites were selected on the basis of their estimated number of patients, their experience in noninterventional studies, the type of site, and their location (to ensure geographical spread within each participating country). All patients received monotherapy with panitumumab $6 \mathrm{mg} / \mathrm{kg}$, once every 2 weeks (Vectibix ${ }^{\circledR}$, Amgen BV, Breda, the Netherlands), at the discretion of the investigator, and in accordance with the European Summary of medicinal Product Characteristics. ${ }^{8}$

The observation period for each patient started on the 1st day of the first monotherapy treatment cycle, and finished at the end of panitumumab therapy, or after a maximum of 18 therapy cycles of 2 weeks' duration each (whichever occurred first). Thus, the maximum observation time was limited to 36 weeks.

\section{Patients}

Patients were adults ( $\geq 18$ years), with histologically or cytologically confirmed KRAS WT mCRC, who had failed prior chemotherapy with 5-fluorouracil, oxaliplatin, and irinotecan. At the time of inclusion, patients were required to have been receiving panitumumab for at least 4 weeks (corresponding to two cycles), to ensure that the decision to prescribe panitumumab was independent of the study. The initial 4 weeks of treatment were documented retrospectively upon inclusion in the study.

\section{Ethics}

The study protocol was approved by the respective country ethical review boards (Bulgaria: Ethics Committee for Multicenter Trials; Slovenia: Republic of Slovenia National Medical Ethics Committee NMEC; Slovakia: Ethics Committee of the Self- Governing Region Kosice; Czech Republic: no submission to ethical committee was necessary at the timepoint the study started; Poland: no submission to ethical committee 
was necessary at the timepoint the study started; Hungary: Medical Research Council, Ethics Committee for Clinical Pharmacology). Written informed consent was gained from all patients in countries where such consent was required for noninterventional, observational studies. The Uniform Requirements for manuscripts submitted to biomedical journals (http://www.icmje.org/) were followed.

\section{Outcome measures}

The incidence and severity of STs (including, but not limited to, acneiform dermatitis, pruritus, erythema, rash, skin exfoliation, paronychia, dry skin, and skin fissures) were assessed according to Common Terminology Criteria for Adverse Events (CTCAE) Version 3.0. Response was assessed at the investigators' discretion at each cycle, at the end of the study, and as best response over the study period. Best response was defined as the maximum response achieved over the study period, and assessed once every four cycles, from cycle 4 to 16 . The disease control rate (DCR) was defined as the percentage of patients with a best response of a complete or partial response, or stable disease. Best response, disease control, tumor response, and freedom from progression (FFP) at the end of therapy were assessed in subgroups of patients according to age ( $<65$ years, 65 to $<80$ years, $\geq 80$ years), Eastern Cooperative Oncology Group (ECOG) performance status, comorbidities, and number of previous chemotherapy courses, as well as by ST grade (maximum grade of any type of ST experienced by each patient).

Data on all adverse drug reactions occurring during panitumumab treatment were collected throughout the study. ECOG performance status and quality of life (rated by the investigator as very good, good, adequate, or bad) were assessed at the end of the study. The KRAS analysis process was also evaluated in terms of time to analysis result, analysis procedure and reporting, and investigators' subjective satisfaction with the process of molecular pathological analysis and its logistics.

\section{Statistical analysis}

As VECTIS was a noninterventional, observational study, no formal hypothesis testing was performed. Assuming a ST rate of $90 \%,{ }^{8,26}$ a sample size of approximately 750 patients was considered adequate.

The full analysis set was defined as all patients who received at least two doses of panitumumab during the observation period. Descriptive statistics were calculated for the study data, and $P$-values generated using two-sided testing. In general, no error adjustment for multiple testing was conducted. Therefore, any $P$-values reported reflect the comparison-related and not the test-related errors. Categorical data were analyzed by Fisher's exact test or the $\chi^{2}$ test. Analyses of differences in quantitative or semiquantitative data were performed using the Wilcoxon test. Multivariate analysis of factors impacting on DCR, tumor response, and FFP were performed using stepwise logistic regression models. The presence of a nonzero correlation between best response and maximum ST grade was tested by the chi-square test for nonzero correlation in ordinal data. The Spearman correlation coefficient and the $P$-value derived from the chi-square test were summarized.

\section{Results}

\section{Patients}

Of the 651 patients included, 632 met eligibility criteria and were included in the full analysis set (Czech Republic,

Table I Baseline characteristics

\begin{tabular}{ll}
\hline & All patients $\mathbf{( N = 6 3 2 )}$ \\
\hline Age (years), mean \pm SD & $62.3 \pm 9.09$ \\
$<65$ years & $358(56.6)$ \\
65 to $<80$ years & $266(42.1)$ \\
$\geq 80$ years & $8(1.3)$ \\
Sex (male) & $408(64.6)$ \\
ECOG performance status & \\
0/I & $585(92.6)$ \\
2 & $47(7.4)$ \\
Concomitant disease & $324(51.3)$ \\
Heart & $152(46.9)$ \\
Liver & $32(9.9)$ \\
Allergy & $25(7.7)$ \\
Lung & $24(7.4)$ \\
Neurological & $18(5.6)$ \\
Renal & $11(3.4)$ \\
Other & $185(57.1)$ \\
Prior surgery (yes) & $584(92.4)$ \\
Prior chemotherapy (yes) & $631(99.8)$ \\
Number of prior chemotherapy regimens & \\
I & $9(1.4)$ \\
2 & $264(4 I .8)$ \\
3 & $202(32.0)$ \\
4 & $103(16.3)$ \\
5 & $38(6.0)$ \\
6 & $12(1.9)$ \\
7 & $3(0.5)$ \\
Prior radiotherapy (yes) & $145(22.9)$ \\
Disease stage at baseline & $129(20.4)$ \\
I-II & $500(79.1)$ \\
III-IV & $3(0.5)$ \\
Unknown & \\
\hline Note Data are & \\
\hline &
\end{tabular}

Note: Data are presented as n (\%) unless otherwise specified.

Abbreviations: ECOG, Eastern Cooperative Oncology Group; SD, standard deviation. 
$\mathrm{n}=357$; Poland, $\mathrm{n}=148$; Hungary, $\mathrm{n}=108$; Slovenia, $\mathrm{n}=8$; Bulgaria, $n=7$; Slovakia, $n=4$ ). Baseline characteristics for the full analysis set are shown in Table 1. Most patients were male ( $n=408 ; 64.6 \%)$, and overall, the mean age was 62.3 years; $358(56.6 \%)$ patients were $<65$ years old. Almost all patients $(n=585 ; 92.6 \%)$ had an ECOG performance status of 0 or 1 , and approximately half of the patients $(n=324$; $51.3 \%$ ) had concomitant diseases (most frequently, heart disease [ $\mathrm{n}=152 ; 46.9 \%])$. Overall, 584 (92.4\%), 631 (99.8\%), and $145(22.9 \%)$ patients had received prior surgery, chemotherapy, or radiotherapy for $\mathrm{mCRC}$, respectively. The mean (standard deviation [SD]) number of prior chemotherapy regimens received was 2.9 (1.06), with a median of 3.0 (range: $0-7)$. Thirty-three patients $(5.2 \%)$ had not received all three specified prior chemotherapy agents; findings from safety and effectiveness sensitivity analyses that excluded these 33 patients were not significantly different than the findings for the full analysis set (data not shown).

\section{Treatment exposure}

Of the 632 patients in the full analysis set, 570 (90.2\%) completed scheduled panitumumab treatment (Figure 1). The mean (SD) number of observed study treatment cycles administered was 9.6 (4.98). Overall, $110(17.4 \%)$ patients completed $>15$ cycles, 159 (25.2\%) completed 11-15 cycles, 195 (30.9\%) completed 6-10 cycles, and 168 (26.6\%) completed five or fewer cycles. Mean panitumumab dosage per patient was 5.9 (0.4) $\mathrm{mg} / \mathrm{kg}$. In 59 (9.3\%) patients, panitumumab treatment was ongoing when 18 cycles was reached. The most common reasons for discontinuation were a change of treatment $(n=13$; $2.1 \%)$, or reasons summarized as "other" ( $\mathrm{n}=25 ; 4.0 \%)$.

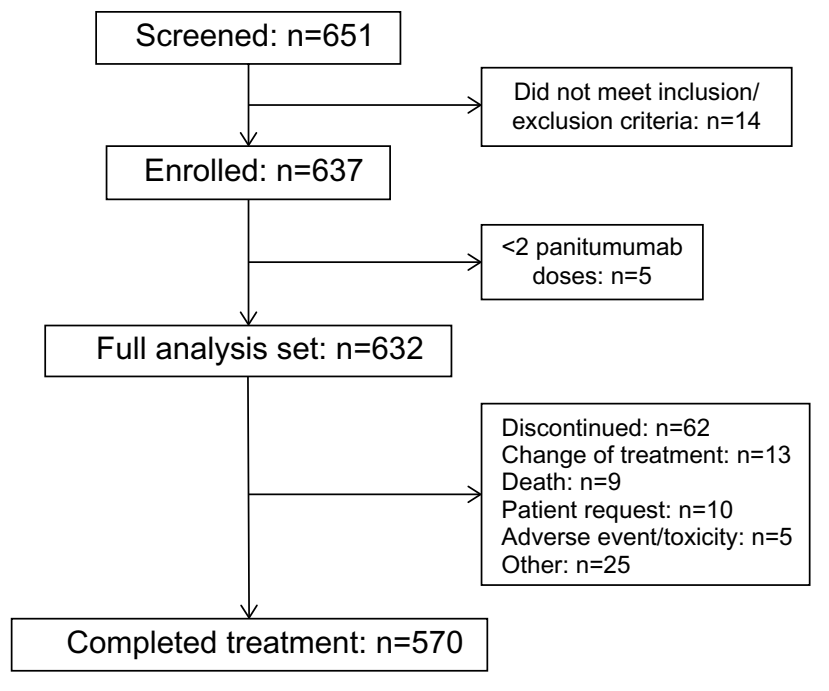

Figure I Patient disposition.
Table 2 The most frequent types of ST adverse drug reactions occurring during the study

\begin{tabular}{|c|c|c|c|c|}
\hline & \multicolumn{4}{|c|}{ All patients $(\mathrm{N}=632)$} \\
\hline & Grade 0 & Grade I & Grade 2 & Grade 3 \\
\hline Any ST ${ }^{a}$ & $99(15.7)$ & $174(27.5)$ & $314(49.7)$ & $45(7.1)$ \\
\hline \multicolumn{5}{|l|}{ Adverse drug reaction ${ }^{\mathrm{b}}$} \\
\hline Rash: acne/acneiform & - & I46 (23.I) & $282(44.6)$ & $38(6.0)$ \\
\hline Dry skin & - & $12(1.9)$ & $18(2.8)$ & $\mathrm{I}(0.2)$ \\
\hline Rash/desquamation & - & $10(1.6)$ & $13(2.1)$ & $3(0.5)$ \\
\hline Nail changes & - & $4(0.6)$ & $12(1.9)$ & $\mathrm{I}(0.2)$ \\
\hline
\end{tabular}

Notes: Data are presented as $\mathrm{n}(\%)$. ${ }^{\mathrm{a}}$ Each patient is counted only once with the maximum grade of skin toxicity (ST) across all cycles. Absence of ST is classified as Grade 0; baccording to Common Terminology Criteria for Adverse Events Version 3.0.

During the study, $62(9.8 \%)$ patients received additional treatment for colorectal cancer. Additionally, 204 (32.3\%) patients received magnesium substitution, and 51 (8.1\%) patients received calcium substitution.

\section{Safety}

During the study, $533(84.3 \%)$ patients reported a total of $782 \mathrm{ST}$ events (Table 2); the incidence rate was 3.3 events per year (95\% confidence interval: 3.10-3.58). Rash (acne/ acneiform) was the most commonly observed ST occurring in $73.7 \%$ of patients overall. ST was grade $1 / 2$ for 488 (77.2\%) patients, and grade 3 for 45 (7.1\%) patients (incidence rate $(I R)=0.21 ; 95 \%$ confidence interval: $0.15-0.28)$. No patients experienced a ST of grade $\geq 4$ during the study. The overall mean (SD) time to first ST was 17.3 (22.2) days. At the end of follow-up, $44.5 \%$ of recorded ST events had not resolved, 29.2\% had resolved, $2.0 \%$ had resolved with sequelae, and $24.2 \%$ were ongoing but improving.

Therapeutic measures for ST were required by 441 patients ( $82.7 \%$ of patients with STs). Of these, topical treatments for skin events were received by 435 (81.6\%) patients, the most common being moisturizing creams and lotions $(\mathrm{n}=317 ; 59.5 \%)$, antibiotics $(\mathrm{n}=187 ; 35.1 \%)$, urea cream/ lotion ( $\mathrm{n}=156 ; 29.3 \%)$, and corticosteroids $(\mathrm{n}=126 ; 23.6 \%)$. Systemic treatment was required by 139 (26.1\%) patients, and comprised antibiotics $(\mathrm{n}=90$ [16.9\%]), antihistamines $(\mathrm{n}=70[13.1 \%])$, and corticosteroids $(\mathrm{n}=13$ [2.4\%]). Topical treatment for nail events was required by $33(6.2 \%)$ patients, the most common being antiseptic measures $(n=22 ; 4.1 \%)$, silver nitrate/antibiotics $(\mathrm{n}=14 ; 2.6 \%)$, and hydrocolloid compounds $(\mathrm{n}=8 ; 1.5 \%)$.

Non-STs were reported by 22 (3.5\%) patients (Table 3 ). During the study, $9(1.4 \%)$ patients died, but no deaths were attributed to panitumumab monotherapy. Dose reductions were required by $77(12.2 \%)$ patients, most commonly for 
Table 3 The most frequent types of nonskin adverse drug reactions occurring during the study

\begin{tabular}{|c|c|c|c|c|}
\hline & \multicolumn{4}{|c|}{ All patients $(\mathrm{N}=632)$} \\
\hline & Overall & Mild & Moderate & Severe \\
\hline Any adverse drug reaction other than ST & $22(3.5)$ & $8(1.3)$ & $10(1.6)$ & $4(0.6)$ \\
\hline \multicolumn{5}{|l|}{ Adverse drug reaction ${ }^{\mathrm{a}}$} \\
\hline Allergic reaction/hypersensitivity (including drug fever) & $4(0.6)$ & 0 & $4(0.6)$ & 0 \\
\hline Diarrhea & $4(0.6)$ & $2(0.3)$ & $2(0.3)$ & 0 \\
\hline Fatigue (asthenia, lethargy, malaise) & $2(0.3)$ & $\mathrm{I}(0.2)$ & $\mathrm{I}(0.2)$ & 0 \\
\hline Hypomagnesemia & $2(0.3)$ & $\mathrm{I}(0.2)$ & 0 & $\mathrm{I}(0.2)$ \\
\hline
\end{tabular}

Notes: Data are presented as n (\%). according to Common Terminology Criteria for Adverse Events Version 3.0. Patients could report more than one adverse event.

Abbreviation: ST, skin toxicity.

STs. Five $(0.8 \%)$ patients discontinued panitumumab as a result of an adverse drug reaction or ST.

\section{Effectiveness}

At the end of treatment, the overall DCR was $58.9 \%$ ( $n=372$; Table 4). A trend toward better outcome was observed with higher ST grade (Table 4). The correlations between DCR, tumor response, FFP, and maximum ST grade are also shown in Table 4. Overall, significant associations were observed between ST grade and DCR $(P=0.0046)$, tumor response $(0.0002)$, best response $(P=0.0009)$, and FFP $(P=0.0084)$. The observed correlations between $\mathrm{ST}$ grade and DCR, tumor response, best response, and FFP, were, however, very low (Rho values: $-0.11,-0.15,-0.14$, and 0.11 , respectively).

In patients with a ST grade of $0 / 1$, the DCR at the end of therapy was $53.8 \%(\mathrm{n}=147 / 273)$ compared with $62.7 \%$ $(n=225 / 359)$ in patients with a ST grade of $2 / 3$. A ST grade of $2 / 3$ vs $0 / 1$ was also associated with improved tumor response $(P=0.0001)$ and FFP $(P=0.0178)$. In addition, patients with fewer prior chemotherapy regimens ( $\leq 3$ vs $>3$ ) showed a nonsignificant trend toward improved tumor response $(P=0.0745)$ and FFP $(P=0.0570)$. Presence of concomitant diseases showed a nonsignificant trend toward lower FFP $(P=0.0507)$. Age, ECOG performance status before start of treatment, disease stage at initial diagnosis, and chemotherapy pretreatment line factors had no significant influence on DCR, tumor response, or FFP at the end of the therapy.

\section{ECOG performance status and quality of life}

Overall, at the end of the study most patients had an ECOG performance status of 0 or $1(n=426 ; 67.4 \%)$. A further $153(24.2 \%)$ patients had an ECOG status of 2, 45 (7.1\%) had an ECOG status of 3, and $5(0.8 \%)$ had an ECOG performance status of 4 .

Further, investigators most frequently rated their patient's quality of life as "good" (n=271; 42.9\%) or "very good" $(\mathrm{n}=173 ; 27.4 \%)$. Patient quality of life was rated as "adequate" $(n=157 ; 24.8 \%)$ or "bad" $(n=31 ; 4.9 \%)$ less frequently.

\section{KRAS testing}

For most patients ( $\mathrm{n}=549 ; 86.9 \%), K R A S$ analysis was conducted on the primary tumor block. In the remaining patients, analysis was performed on samples obtained through

Table 4 Treatment response at the end of therapy overall and by severity of ST

\begin{tabular}{|c|c|c|c|c|c|c|c|}
\hline & \multicolumn{7}{|c|}{ Maximum grade of $\mathbf{S T}^{\mathbf{a}}$} \\
\hline & $\begin{array}{l}\text { Overall } \\
(\mathrm{N}=632)\end{array}$ & $\begin{array}{l}\text { Grade } 0 \\
(n=99)\end{array}$ & $\begin{array}{l}\text { Grade I } \\
(n=174)\end{array}$ & $\begin{array}{l}\text { Grade } 2 \\
(n=3 \mid 4)\end{array}$ & $\begin{array}{l}\text { Grade } 3 \\
(n=45)\end{array}$ & $\begin{array}{l}\text { Spearman correlation } \\
\text { coefficient }\end{array}$ & $P$-value \\
\hline Best response & & & & & & -0.14 & 0.0009 \\
\hline Complete response & $6(0.9)$ & 0 & $2(0.3)$ & $3(0.5)$ & $\mathrm{I}(0.2)$ & & \\
\hline Partial response & $60(9.5)$ & $2(0.3)$ & $9(1.4)$ & $45(7.1)$ & $4(0.6)$ & & \\
\hline Stable disease & $306(48.4)$ & $44(7.0)$ & $90(14.2)$ & $147(23.3)$ & $25(4.0)$ & & \\
\hline Progressive disease & $248(39.2)$ & $47(7.4)$ & $7 I(I I .2)$ & $117(18.5)$ & $13(2.1)$ & & \\
\hline Not assessable & $12(1.9)$ & $6(0.9)$ & $2(0.3)$ & $2(0.3)$ & $2(0.3)$ & & \\
\hline Disease control rate ${ }^{b}$ & 372 (58.9) & $46(7.3)$ & $101(16.0)$ & $195(30.9)$ & $30(4.7)$ & -0.11 & 0.0046 \\
\hline Tumor response $^{c}$ & $66(10.4)$ & $2(0.3)$ & II (I.7) & $48(7.6)$ & $5(0.8)$ & -0.15 & 0.0002 \\
\hline Freedom from progression ${ }^{d}$ & $115(18.2)$ & $13(2.1)$ & $24(3.8)$ & $66(10.4)$ & $12(1.9)$ & 0.11 & 0.0084 \\
\hline
\end{tabular}

Notes: Data are presented as $\mathrm{n}(\%)$ unless otherwise specified. ${ }^{\mathrm{A}} \mathrm{A}$ patient with no recorded ST reactions during the observation period is considered to have Grade 0 ; ${ }^{\mathrm{b}}$ defined as complete response, partial response, or stable disease at end of therapy; 'defined as complete response or partial response at end of therapy; ${ }^{\mathrm{d}}$ at end of therapy. Abbreviation: ST, skin toxicity. 
resection of metastases $(\mathrm{n}=49 ; 7.8 \%)$, biopsy of metastases $(n=21 ; 3.3 \%)$, or another biopsy $(n=13 ; 2.1 \%)$. Problems with obtaining a specimen for analysis were reported for two $(0.3 \%)$ patients. Sequencing $(n=343 ; 54.3 \%)$ was the most common method used to determine tumor $K R A S$ status, followed by the DxS test $(n=200 ; 31.6 \%)$ and pyrosequencing $(\mathrm{n}=5 ; 0.8 \%)$. Other methods of analysis were used to determine $K R A S$ status in $84(13.3 \%)$ patients.

Overall, the mean (SD) time required to obtain $K R A S$ results was 18.2 (10.53) days. Satisfaction with the speed of testing ( $\mathrm{n}=511 ; 80.9 \%)$, organization of sample dispatch $(n=591 ; 93.5 \%)$, clarity of final report $(n=613 ; 97.0 \%)$, and cooperation with the responsible institution $(n=604 ; 95.6 \%)$ were mostly rated as "good" or "very good".

\section{Discussion}

The VECTIS observational study was conducted to collect information on the use of panitumumab monotherapy when administered in accordance with the European Summary of medicinal Product Characteristics, in daily practice in patients with $K R A S$ WT mCRC. The study was also designed to collect data specifically related to the occurrence of STs and their management in clinical practice.

Compared with the populations included in controlled Phase II and III studies of panitumumab monotherapy as third- or higher-line anticancer treatment, ${ }^{27-29}$ the population of this clinical practice study was of a similar age and sex distribution. In VECTIS, more patients had an ECOG performance status of 1 or higher than either the Phase III study $^{27}$ or the Japanese Phase II study, ${ }^{29}$ but proportions were similar to those reported in the US study. ${ }^{28}$ More patients in VECTIS were receiving the third- or higher line of mCRC treatment than in some of the other monotherapy studies. ${ }^{27,29}$

The results of VECTIS show that the use of panitumumab monotherapy in heavily pretreated mCRC patients was associated with a similar effectiveness and safety profile to that which would be expected, based on published clinical trial data in this setting. ${ }^{27-29}$ At the end of treatment, 1\% of patients had a complete response (CR), whereas no CRs were observed in the other studies. A partial response (PR) was observed in $10 \%$ of patients in VECTIS, which is in line with the Phase II and III studies leading to the approval of panitumumab. ${ }^{27-29}$ In contrast, the recent Phase III ASPECCT trial reported a PR rate of $22 \%$ during panitumumab treatment. ${ }^{30}$ However, more patients in VECTIS than in the comparable monotherapy studies had SD (48\% in VECTIS vs $27 \%-33 \%$ in the other studies). ${ }^{27-29}$ Importantly, it is known from previous studies that disease stabilization is a clinically relevant response in the chemotherapy-refractory-mCRC setting. ${ }^{27,31,32}$ Overall, a tumor response (CR + PR) was observed in $10 \%$ of patients in VECTIS, and disease control (CR + PR $+\mathrm{SD}$ ) was observed in $59 \%$ of patients, compared with tumor response rates of $9 \%-14 \%$ (PR only), and DCRs of $37 \%-47 \%$ reported in the literature. ${ }^{27-29}$ The progressive disease rate was $39 \%$ in VECTIS, compared with $40 \%-50 \%$ reported in the literature. $^{28,29}$

The influence of patient age, ECOG performance status, comorbidities, prior treatment, and ST grade on outcomes during panitumumab treatment was also investigated in the present study. Overall, there were associations between ST grade and DCR, tumor response, and FFP, although these correlations could not be confirmed in each individual participating country. A significant correlation between efficacy and maximum ST grade has previously been reported, ${ }^{23-25}$ but has yet to be validated, and should not be used as a surrogate clinical marker for response. Absence of concomitant disease and a lower number of prior chemotherapy regimens also appeared to be associated with improved tumor response and FFP, although the trends were not statistically significant. In contrast, age and ECOG performance status did not appear to influence the effectiveness of panitumumab treatment.

Mild to moderate ST is a common side effect of EGFRtargeted agents. ${ }^{28}$ Among the patients treated in VECTIS, $84 \%$ experienced ST overall, with $28 \%$ experiencing grade 1 and $57 \%$ experiencing grade $\geq 2$ events. No patients experienced grade $4 / 5 \mathrm{ST}$ during the study. Of the patients with ST, $83 \%$ received a therapeutic measure for these events, with $82 \%$ receiving topical skin treatment, $6 \%$ receiving topical nail treatment, and 26\% receiving systemic treatment. In the present study, ST was generally manageable, with $29 \%$ of patients experiencing symptom resolution and $24 \%$ showing symptom improvement by study end. In a study evaluating the benefits of preemptive vs reactive treatment of panitumumab-related STs (STEPP), ${ }^{33} 29 \%$ of preemptively treated and $62 \%$ of reactively treated patients experienced grade $\geq 2$ STs. In VECTIS, investigators were not asked specifically if they had provided ST treatment preemptively or reactively, but the incidence of STs and the proportion of patients needing systemic treatment suggest the latter. Prophylactically administering skin treatments parallel to panitumumab therapy may reduce the incidence of moderate to severe ST events.

Limitations of the present study include its open-label, uncontrolled, noninterventional study design (although such 
designs are important for evaluating the use of treatments in routine clinical practice), the small numbers of patients included from some countries, and the maximum of 18 panitumumab treatment cycles. There are also important differences between routine clinical practice and interventional clinical trials. For example, the patient population in VECTIS was unselected and included all the patients who were eligible for panitumumab monotherapy, as per licensed indication. However, perhaps the most important limitation pertaining to our observational study design was the lack of centralized assessment of KRAS status and tumor response. Response was assessed by the investigators using the methods available at their centers, which may explain some of the differences seen between our study and the published Phase II and III trials. In addition, recent analyses show that $R A S \mathrm{WT}$ status, as determined in an expanded $R A S$ analysis including $K R A S$ and $N R A S$ exons $2-4$, better predicts those patients most likely to benefit from panitumumab therapy. Studies have found that approximately $10 \%-20 \%$ of $K R A S$ exon 2 WT tumors harbor other clinically relevant $R A S$ mutations. ${ }^{15-21}$ Therefore, it would be expected that data from VECTIS would have reflected the improved benefit/risk profile of panitumumab had the study population been limited to patients with $R A S$ WT tumors. At the time the study was conducted, however, $R A S$ testing was limited to exon 2 of KRAS.

\section{Conclusion}

Panitumumab showed an acceptable effectiveness and safety profile when used as monotherapy for patients with heavily pretreated KRAS WT mCRC. Treatment was generally well tolerated, with grade $1 / 2 \mathrm{ST}$ being the most common adverse drug reaction reported; only $7 \%$ of patients experienced grade $3 \mathrm{ST}$. The study outcomes represent a valuable contribution to existing knowledge of STs associated with panitumumab, the time taken to develop such toxicities, and the practical aspects of the KRAS analysis process.

\section{Acknowledgments}

The authors would like to thank the VECTIS study investigators for participating and enrolling patients into the study. Medical writing assistance was provided by Daniel Booth, PhD, and Dawn Batty, PhD (Bioscript Medical, Macclesfield, UK), funded by Amgen (Europe) $\mathrm{GmbH}$, and Margit Hemetsberger, $\mathrm{PhD}$ (Hemetsberger Medical Services, Vienna, Austria), funded by Amgen CEE Head Office. Masaryk Memorial Cancer Institute obtained institutional support by MH CZ - DRO (MMCI 00209805). This study was funded by Amgen CEE Head Office and participating countries. The study sponsors (Amgen Poland, Amgen Czech Republic, and Amgen CEE Head Office) were involved in the study design, in the collection, analysis, and interpretation of data, and in the writing of the manuscript. The decision to submit the manuscript for publication was taken jointly by the authors.

\section{Author contributions}

RL, WR, BP, ZM, and EP contributed to the collection, analysis, and interpretation of data, and the writing of the manuscript. RL contributed to the study design. LK contributed to the interpretation of data and the writing of the manuscript.

\section{Disclosure}

RL reports personal fees and nonfinancial support from Amgen during the conduct of the study, and personal fees from Sanofi, Roche, and Bristol-Myers-Squibb, unrelated to the submitted work. WR, BP, ZM, and EP have no conflicts of interest to disclose. LK is an employee of Amgen and holds Amgen stock.

\section{References}

1. Ferlay J, Steliarova-Foucher E, Lortet-Tieulent J, et al. Cancer incidence and mortality patterns in Europe: estimates for 40 countries in 2012. Eur J Cancer. 2013;49(6):1374-1403.

2. Patel GS, Karapetis CS. Personalized treatment for advanced colorectal cancer: KRAS and beyond. Cancer Manag Res. 2013;5:387-400.

3. Chu E. An update on the current and emerging targeted agents in metastatic colorectal cancer. Clin Colorectal Cancer. 2012; 11(1):1-13.

4. Prenen H, Vecchione L, Van Cutsem E. Role of targeted agents in metastatic colorectal cancer. Target Oncol. 2013;8(2):83-96.

5. Foubert F, Matysiak-Budnik T, Touchefeu Y. Options for metastatic colorectal cancer beyond the second line of treatment. Dig Liver Dis. 2014;46(2):105-112.

6. Cohenuram M, Saif MW. Panitumumab the first fully human monoclonal antibody: from the bench to the clinic. Anticancer Drugs. 2007;18(1): 7-15.

7. Argiles G, Dienstmann R, Elez E, Tabernero J. Panitumumab: a summary of clinical development in colorectal cancer and future directions. Future Oncol. 2012;8(4):373-389.

8. Vectibix ${ }^{\circledR}$ [Summary of medicinal Product Characteristics]. London, UK: European Medicines Agency; 2014. Available from: http://www. ema.europa.eu/ema/index.jsp?curl=pages/medicines/human/ medicines/000741/human_med_001128.jsp\&mid=WC0b01ac058001 d124. Accessed July 14, 2015.

9. Foon KA, Yang XD, Weiner LM, et al. Preclinical and clinical evaluations of ABX-EGF, a fully human anti-epidermal growth factor receptor antibody. Int J Radiat Oncol Biol Phys. 2004;58(3):984-990.

10. Amado RG, Wolf M, Peeters M, et al. Wild-type KRAS is required for panitumumab efficacy in patients with metastatic colorectal cancer. J Clin Oncol. 2008;26(10):1626-1634.

11. Douillard JY, Siena S, Cassidy J, et al. Randomized, phase III trial of panitumumab with infusional fluorouracil, leucovorin, and oxaliplatin (FOLFOX4) versus FOLFOX4 alone as first-line treatment in patients with previously untreated metastatic colorectal cancer: the PRIME study. J Clin Oncol. 2010;28(31):4697-4705. 
12. Peeters M, Price TJ, Cervantes A, et al. Randomized phase III study of panitumumab with fluorouracil, leucovorin, and irinotecan (FOLFIRI) compared with FOLFIRI alone as second-line treatment in patients with metastatic colorectal cancer. J Clin Oncol. 2010;28(31): 4706-4713.

13. Van Cutsem E, Kohne CH, Hitre E, et al. Cetuximab and chemotherapy as initial treatment for metastatic colorectal cancer. $N$ Engl J Med. 2009;360(14):1408-1417.

14. Van Cutsem E, Nordlinger B, Cervantes A; ESMO Guidelines Working Group. Advanced colorectal cancer: ESMO Clinical Practice Guidelines for treatment. Ann Oncol. 2010;21(Suppl 5):v93-v97.

15. Peeters M, Oliner KS, Parker A, et al. Massively parallel tumor multigene sequencing to evaluate response to panitumumab in a randomized phase III study of metastatic colorectal cancer. Clin Cancer Res. 2013;19(7):1902.

16. Peeters M, Douillard JY, Van Cutsem E, et al. Mutant KRAS codon 12 and 13 alleles in patients with metastatic colorectal cancer: assessment as prognostic and predictive biomarkers of response to panitumumab. J Clin Oncol. 2013;31(6):759-765.

17. De Roock W, Jonker DJ, Di Nicolantonio F, et al. Association of KRAS p.G13D mutation with outcome in patients with chemotherapyrefractory metastatic colorectal cancer treated with cetuximab. JAMA. 2010;304(16):1812-1820.

18. De Roock W, Claes B, Bernasconi D, et al. Effects of KRAS, BRAF, NRAS, and PIK3CA mutations on the efficacy of cetuximab plus chemotherapy in chemotherapy-refractory metastatic colorectal cancer: a retrospective consortium analysis. Lancet Oncol. 2010;11(8): 753-762.

19. Andre T, Blons H, Mabro M, et al. Panitumumab combined with irinotecan for patients with KRAS wild-type metastatic colorectal cancer refractory to standard chemotherapy: a GERCOR efficacy, tolerance, and translational molecular study. Ann Oncol. 2013;24(2):412-419.

20. Loupakis F, Ruzzo A, Cremolini C, et al. KRAS codon 61, 146 and BRAF mutations predict resistance to cetuximab plus irinotecan in KRAS codon 12 and 13 wild-type metastatic colorectal cancer. $\mathrm{Br} J$ Cancer. 2009;101(4):715.

21. Schwartzberg LS, Rivera F, Karthaus M, et al. PEAK: a randomized, multicenter phase II study of panitumumab plus modified fluorouracil, leucovorin, and oxaliplatin (mFOLFOX6) or bevacizumab plus mFOLFOX6 in patients with previously untreated, unresectable, wild-type KRAS exon 2 metastatic colorectal cancer. J Clin Oncol. 2014;32(21):2240-2247.

22. Erbitux ${ }^{\circledR}$ [Summary of medicinal Product Characteristcs]. London, UK: European Medicines Agency; 2014. Available from: http:// www.ema.europa.eu/ema/index.jsp?curl=pages/medicines/human/ medicines/000558/human_med_000769.jsp\&mid=WC0b01ac058 001d124. Accessed July 14, 2015.
23. Peeters M, Siena S, Van Cutsem E, et al. Association of progression-free survival, overall survival, and patient-reported outcomes by skin toxicity and KRAS status in patients receiving panitumumab monotherapy. Cancer. 2009;115(7):1544-1554.

24. Douillard JY, Siena S, Tabernero J, et al. Final skin toxicity (ST) and patient-reported outcomes (PRO) results from PRIME: a randomized phase III study of panitumumab (pmab) plus FOLFOX4 (CT) for first-line metastatic colorectal cancer (mCRC). J Clin Oncol. 2012;30(Suppl 4):Abstract 531

25. Price TJ, Sobrero AF, Wilson G, et al. Randomized, open-label, phase III study of panitumumab (pmab) with FOLFIRI versus FOLFIRI alone as second-line treatment (tx) in patients (pts) with metastatic colorectal cancer (mCRC): efficacy by skin toxicity (ST). J Clin Oncol. 2010;28(Suppl 15):3529.

26. Peuvrel L, Bachmeyer C, Reguiai Z, et al. Semiology of skin toxicity associated with epidermal growth factor receptor (EGFR) inhibitors. Support Care Cancer. 2012;20(5):909-921.

27. Van Cutsem E, Peeters M, Siena S, et al. Open-label phase III trial of panitumumab plus best supportive care compared with best supportive care alone in patients with chemotherapy-refractory metastatic colorectal cancer. J Clin Oncol. 2007;25(13):1658-1664.

28. Hecht JR, Patnaik A, Berlin J, et al. Panitumumab monotherapy in patients with previously treated metastatic colorectal cancer. Cancer. 2007;110(5):980

29. Muro K, Yoshino T, Doi T, et al. A phase 2 clinical trial of panitumumab monotherapy in Japanese patients with metastatic colorectal cancer. Jpn J Clin Oncol. 2009;39(5):321-326.

30. Price TJ, Peeters M, Kim TW, et al. Panitumumab versus cetuximab in patients with chemotherapy-refractory wild-type KRAS exon 2 metastatic colorectal cancer (ASPECCT): a randomised, multicentre, open-label, non- inferiority phase 3 study. Lancet Oncol. 2014;15(6): 569-579.

31. Rougier P, Van Cutsem E, Bajetta E, et al. Randomised trial of irinotecan versus fluorouracil by continuous infusion after fluorouracil failure in patients with metastatic colorectal cancer. Lancet. 1998; 352(9138):1407-1412.

32. Cunningham D, Humblet Y, Siena S, et al. Cetuximab monotherapy and cetuximab plus irinotecan in irinotecan-refractory metastatic colorectal cancer. N Engl J Med. 2004;351(4):337-345.

33. Lacouture ME, Mitchell EP, Piperdi B, et al. Skin toxicity evaluation protocol with panitumumab (STEPP), a phase II, open-label, randomized trial evaluating the impact of a pre-emptive skin treatment regimen on skin toxicities and quality of life in patients with metastatic colorectal cancer. J Clin Oncol. 2010;28(8):1351-1357.
Cancer Management and Research

\section{Publish your work in this journal}

Cancer Management and Research is an international, peer-reviewed open access journal focusing on cancer research and the optimal use of preventative and integrated treatment interventions to achieve improved outcomes, enhanced survival and quality of life for the cancer patient. The journal welcomes original research, clinical \& epidemiological

\section{Dovepress}

studies, reviews \& evaluations, guidelines, expert opinion \& commentary, case reports \& extended reports. The manuscript management system is completely online and includes a very quick and fair peerreview system, which is all easy to use. Visit http://www.dovepress.com/ testimonials.php to read real quotes from published authors. 